\title{
Diffusion of Water and Diatomic Oxygen in Poly(3-hexylthiophene) Melt: A Molecular Dynamics Simulation Study
}

\author{
Julia Deitz, Yeneneh Yimer, and Mesfin Tsige \\ Department of Polymer Science \\ University of Akron \\ 302 Buchtel Common \\ Akron, Ohio 44325 USA
}

Received: August 4, 2011 Accepted: November 28, 2011

\begin{abstract}
Diffusion behavior of water, diatomic oxygen, and a mixture of both into a poly(3hexylthiophene) $[\mathrm{P} 3 \mathrm{HT}]$ melt were investigated using Molecular Dynamics Simulation. Once simulations were complete, the data was analyzed to determine the diffusion coefficient of those molecules in P3HT using Fick's law. The diffusion coefficient values were then plotted as a function of concentration and temperature to determine if trends existed. For both water and oxygen, no dependence was observed of the diffusion coefficient on concentration and temperature for the ranges studied. However, a variation in the diffusion coefficient on concentration was observed due to the expected inhomogeneity of the $\mathrm{P} 3 \mathrm{HT}$ melt. In the presence of $\mathrm{O}_{2}$, the diffusion of $\mathrm{H}_{2} \mathrm{O}$ decreased significantly by a factor between four and five, while in the presence of $\mathrm{H}_{2} \mathrm{O}$, the diffusion of $\mathrm{O}_{2}$ slightly decreased.
\end{abstract}

\section{INTRODUCTION}

For the next fifty years, energy is thought to be the number one issue humanity will face [1]. Given this heightened concern, renewable sources are more important than ever. Solar technology offers a long term solution to this issue, but also presents problems of its own. Silicon panels dominate the solar market, but are brittle and expensive. On the other hand, polymer based solar cells are cheap, flexible, lightweight, and easily processed and are thus of both major technological importance and scientific interest. Among polymer devices, Poly(3-hexylthiophene)[P3HT] is a particularly good candidate because it offers good conductivity and solubility. Unfortunately, polymer solar devices, including $\mathrm{P} 3 \mathrm{HT}$, face stability concerns with lighting, heating, $\mathrm{H}_{2} \mathrm{O}$, and $\mathrm{O}_{2}$ causing degradation.
To address the stability concerns of the P3HT solar device, a basic understanding of what is occurring in degradation needs to be reached. Specifically, the degradation that occurs with water and oxygen diffusion in $\mathrm{P} 3 \mathrm{HT}$ is important to explore because they are an essential part of the environment that the P3HT devices operate in. This work sought to address the diffusion that occurs with $\mathrm{H}_{2} \mathrm{O}$ and $\mathrm{O}_{2}$ in $\mathrm{P} 3 \mathrm{HT}$. Using Molecular Dynamics Simulation, the diffusion of water and oxygen into a system of P3HT molecules was simulated and analyzed.

For comparison purposes, experimental values were sought after. No theoretical work regarding the diffusion of $\mathrm{H}_{2} \mathrm{O}$ and $\mathrm{O}_{2}$ in P3HT has been found. The diffusion coefficient of $\mathrm{O}_{2}$ into a system of P3HT was found to be $3 \times 10^{-8} \mathrm{~cm}^{2} \mathrm{~s}^{-1}$ at $295 \mathrm{~K}$ in one experiment [2]. The same experiment found that humidity only increased this rate and did very little on its 


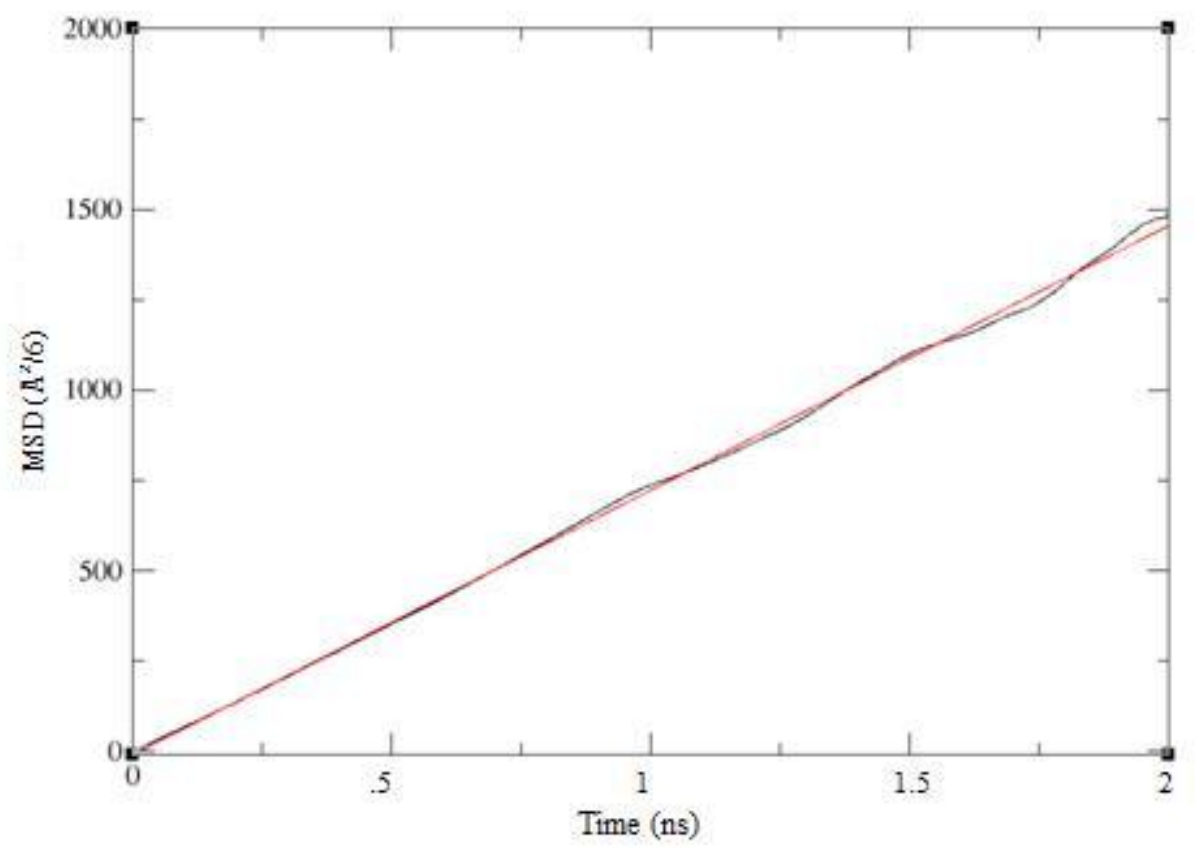

Figure 1. MSD vs. Time at $590 \mathrm{~K}$ with 20 Molecules of $\mathrm{H} 2 \mathrm{O}$ in $\mathrm{P} 3 \mathrm{HT}$.

own. The mentioned experiment was done for crystalline $\mathrm{P} 3 \mathrm{HT}$, but for the simulation in this work, the P3HT was in melt form because appropriate force field for simulation of $\mathrm{P} 3 \mathrm{HT}$ is already established for that state.

\section{METHODOLOGY}

\section{a. Simulation Methods}

For P3HT, we used optimized parameter for liquid simulation-all atom (OPLS-AA) force field [3] which was used to simulate bulk properties of melt P3HT. For the water molecule, we used the SPC/E water model [4]. For diatomic oxygen, we used OPLS-AA force field [5] which was used to study the diffusion of oxygen molecules in different polymeric materials. The P3HT chain was built in regioregular fashion. In this study the P3HT system consists of $100 \mathrm{P} 3 \mathrm{HT}$ chains and within a chain there are 10 monomers (3hexylthiophene). Initially, the P3HT chain was placed randomly in simulation box. The equilibration of the P3HT system using molecular dynamics simulation (MD) was done in two steps. First, the MD simulation at constant temperature and pressure (NPT) was done for a period of $5 \mathrm{~ns}$ to equilibrate the P3HT to its liquid density at $550 \mathrm{~K}$. The final box dimension was $L_{x}=L_{y}=L_{z}=67.987 \AA$ and for all the simulations this box size was kept constant. Secondly, the system was further equilibrated at constant volume and temperature (NVT) at $550 \mathrm{~K}$ for $5 \mathrm{~ns}$.

To study the diffusion of water, diatomic oxygen, and mixture of both in P3HT melt, different number of molecules were inserted into the pre-equilibrated $\mathrm{P} 3 \mathrm{HT}$ melt. The numbers of molecules considered are: 1 - 20 in a step of 5 molecules for water, 5, 10 and 20 molecules for diatomic oxygen and for the mixture case, 20 water molecules and 20 diatomic oxygen molecules. For all cases we run NVT ensembles for $2.5 \mathrm{~ns}$ and the last 2 ns were used for analysis. For water and diatomic oxygen molecules the simulations were done in temperature ranges of $540 \mathrm{~K}$ to 640 $\mathrm{K}$, and for the mixture cases simulations at $550 \mathrm{~K}, 575 \mathrm{~K}$ and $600 \mathrm{~K}$ were done. All the molecular dynamics simulations were carried out using LAMMPS molecular dynamic package [6]. The equations of motion were integrated using Velocity-Verlet algorithm using a time step 1 fs. For both NPT and NVT ensembles calculations a Nose-Hoover thermostat with a damping 


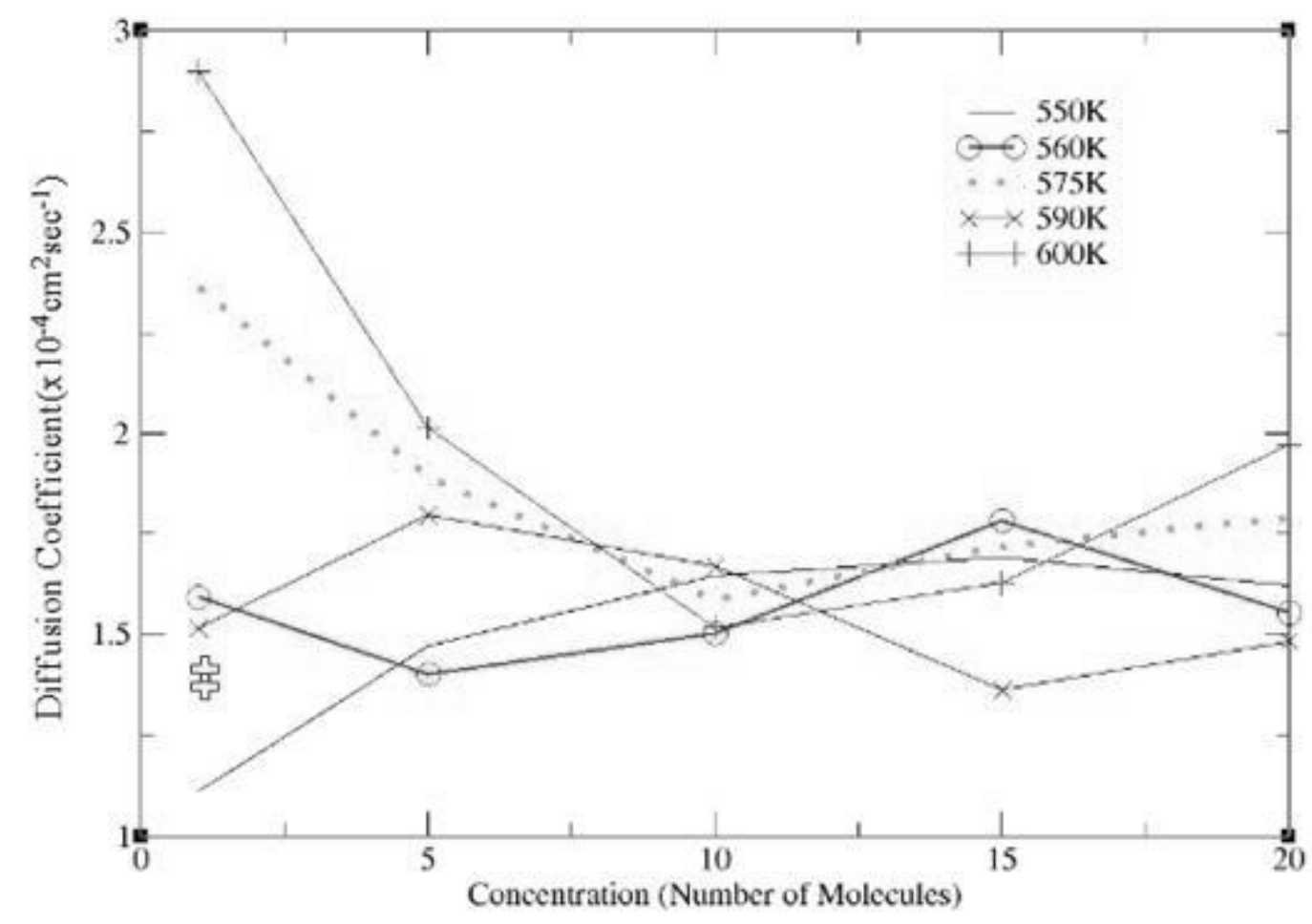

Figure 2. Diffusion coefficient of $\mathrm{H}_{2} \mathrm{O}$ in $\mathrm{P} 3 \mathrm{HT}$ vs. concentration.

time of 100 fs was used to maintain temperature of the system. In addition, to control the pressure for the NPT ensemble calculations, we employed a Nose-Hoover barostat with a 1000 fs damping constant and the pressure was kept at $1 \mathrm{~atm}$. For the non-bond interaction the cutoff radius were $12 \AA$ and the long-range coulomb interaction (beyond the cutoff radius) were calculated using a particle-particle-mesh Ewald algorithm.

b. Determination of the Diffusion Coefficient

The position of each atom over time was given from molecular dynamics simulation. It was then possible to determine the diffusion coefficient based on the Einstein equation:

$$
M S D(t)=\left\langle R^{2}(t)\right\rangle=\Sigma\left\langle|r(t+\tau)-r(\tau)|^{2}\right\rangle
$$

$M S D$ represents the mean square displacement, and $r$ represents the position of a particular molecule. Time is simply signified by $t$, and $\tau$ is a reference time. Once this was found, the diffusion coefficient could be calculated using:

$$
\left\langle R^{2}(t)\right\rangle=6 D t
$$

where $D$ is the diffusion coefficient. The diffusion coefficient was calculated by averaging over molecules and time. A graph of MSD/6 vs. time would yield the diffusion coefficient as the slope.

A best fit line was then made to cross the origin and capture early behavior of diffusion. Diffusion coefficients were also found to fit different time frames, and the trends remained the same. For consistency purposes, all values used in this paper were taken from the beginning. Figure 1 is an example of this and was fit to match the range from 0 to .6 nanoseconds but it matches decently up to 2 nanoseconds. In figure 1 , the linear, red line is the best fit line, while the black, less linear line represents the data.

\section{RESULTS AND DISCUSSION:}

\section{a. Water Diffusion in P3HT}

The diffusion coefficients for water in P3HT were tabulated and then analyzed for behavior. They were graphed as a 


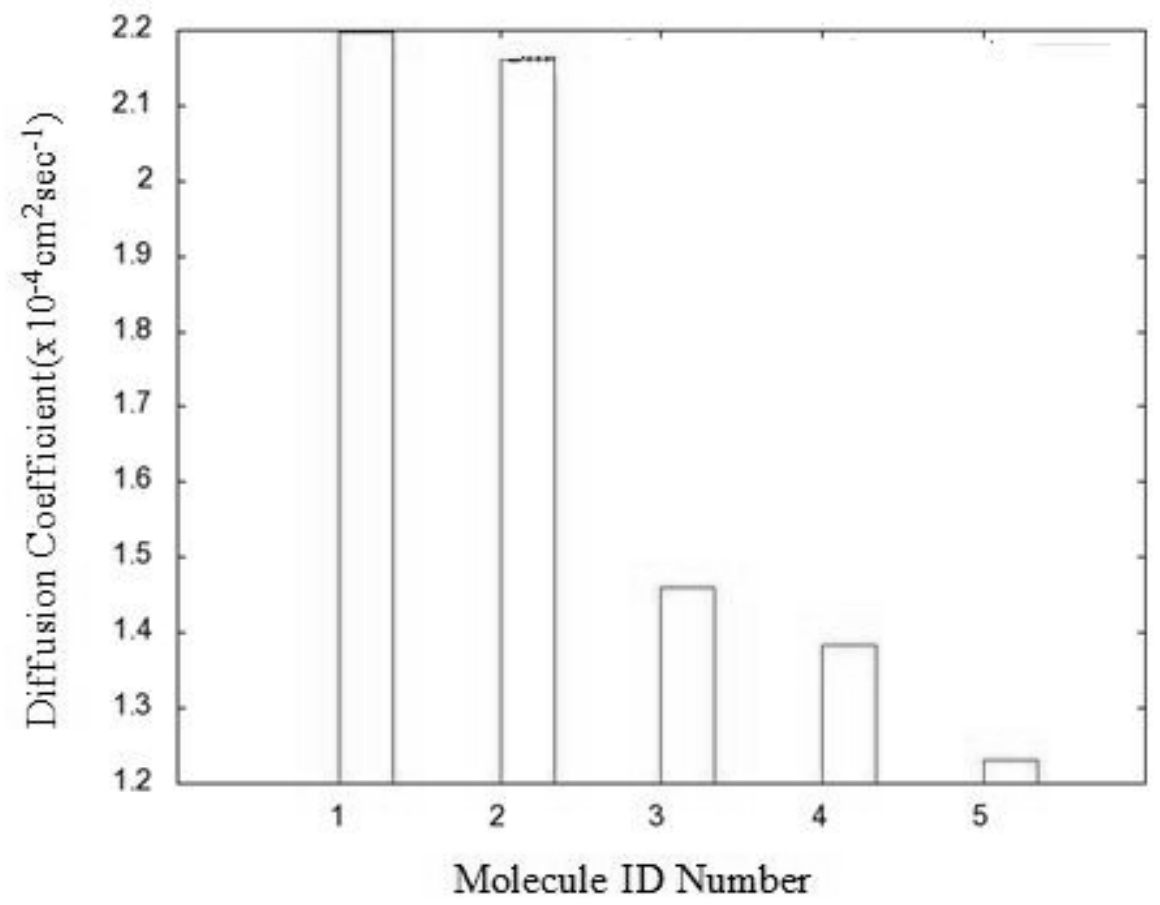

Figure 3. Diffusion coefficient values for individual water molecules at $550 \mathrm{~K}$ in the $5 \mathrm{H}_{2} \mathrm{O}$ in $\mathrm{P} 3 \mathrm{HT}$ system

function of concentration and also of temperature to determine if dependence existed. First, the diffusion coefficient was looked at as a function of water concentration in the P3HT system, as shown in figure 2. As seen in figure 2, for one $\mathrm{H}_{2} \mathrm{O}$ molecule there is large variation in the diffusion, but for the other values the diffusion converges to a constant value. In attempts to gain insight on what was happeining with the diffusion, new initial positions were used for the concentration of one molecule. Also, the diffusion coefficients for individual water molecules in the 5 molecule system were examined.

To test dependence, two new intial positions for the one molecule concentration were simulated at $550 \mathrm{~K}$. The two brown crosses in Figure 2 represent the diffusion coefficient values found for the two new positions used. As shown, the additional values differed from the original one. Dependence on concentration was investigated by examining the difference in the diffusion coefficients of the individual molecules of a higher concentration system. The five molecule system was analyzed, and the results are shown in Figure 3 . As indicated in figure 3 , the disparity amoungst individual molecules in the system was significant; the coefficients varied by about a factor of two. The inconsistency illustrated in Figure 3 was also seen in the diffusion coefficients for the concentration of one molecule in figure 2. The inhomogentiy of the P3HT caused the water molecules to diffuse differently at different positions. For higher concentrations, the diffusion values converged because of the averaging done over molecules. The water diffusion into P3HT was not dependent on concentration within the range of our study.

An explanation as to why concentration dependence did not exist was that clustering of the water molecules was not occuring. If the water molecules formed clusters during the simulation, then the diffusion coefficient would be a function of concentration. Visual Molecular Dynamics(VMD) [7] was used to visualize if in fact the water molecules were clustering or not. The water molecules did not show clustering; also indicative of no dependence on concentration. This is shown in figures 4. The larger spherical molecules represent the water amoungst the P3HT molecules. The 

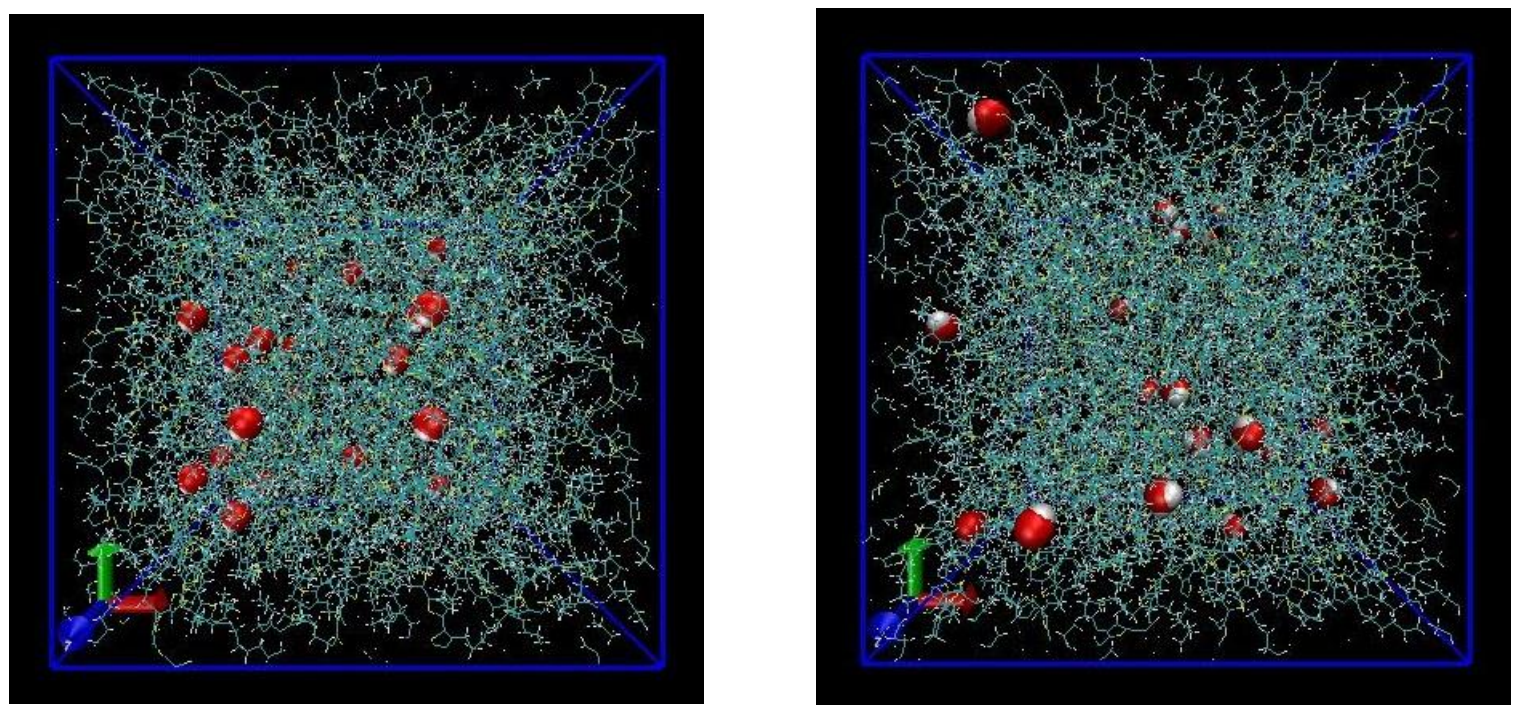

Figure 4. $20 \mathrm{H}_{2} \mathrm{O}$ molecules in $\mathrm{P} 3 \mathrm{HT}$ melt at $550 \mathrm{~K}$ at $\mathrm{t}=0 \mathrm{~ns}$ (left) and $\mathrm{t}=2.5 \mathrm{~ns}$ (right). $\mathrm{P} 3 \mathrm{HT}$ molecules are shown in bond representation; atoms in water molecules are represented by van der Waals spheres.

VMD graphics showed the same pattern in all concentrations and all temperatures. The water molecules did not cluster together. However, there could be a critical water concentration limit beyond which the water molecules in the P3HT system may start to form clusters, but this is beyond the goal of the present work.

As figure 5 depicts, the diffusion coefficient was then analyzed as a function of temperature. A dependence on temperature could not be seen in the temperature window used in figure 5 . To further investigate this, additional values for diffusion coefficient were found for the twenty molecule system at $540 \mathrm{~K}, 610 \mathrm{~K}$, $620 \mathrm{~K}, 630 \mathrm{~K}$, and $640 \mathrm{~K}$ to examine if any temperature dependence could be seen at other temperatures.

For the twenty molecule line with a wider window of temperatures, the diffusion coefficient continued to show no dependence on temperature. The results demonstrated that even up to $640 \mathrm{~K}$, the diffusion of water into $\mathrm{P} 3 \mathrm{HT}$ was not a function on temperature even though it was expected that the diffusion would increase with temperature. Possibly, after a certain point, the diffusion of water in the $\mathrm{P} 3 \mathrm{HT}$ system plauteaued, making it difficult to notice behaviors with changing temperature. This could be a result of the slow molecular dynamics of $\mathrm{P} 3 \mathrm{HT}$ changing little in the temperature window used. Also, potentially the time window of 2.5 nanoseconds did not allow much change in the P3HT dynamics either. If the dynamics of the P3HT were unchanging for the time and temperature lapses used, then diffusion may not show strong dependence on temperature, which is observed in the present study.

\section{b. Diatomic Oxygen Diffusion in P3HT}

Diffusion coefficients of $\mathrm{O}_{2}$ in $\mathrm{P} 3 \mathrm{HT}$ were also calculated. Dependence on concentration and temperature were then looked for. First, the diffusion coefficient values were examined as a function of concentration, shown in figure 6. Similar to water diffusion, the diffusion coefficient values varied significantly at the concentration of one molecule, but converged to a constant value as concentration increased. This happened because of the averaging of the diffusion coefficient over molecule when calculating the MSD. With one, individual molecule, no averaging over molecules could occur so more variation is seen. These findings demostrate that diffusion of oxygen in P3HT is not a function of concentration within the range of our study. Any variation is due to the inhomogenity of the P3HT melt. 


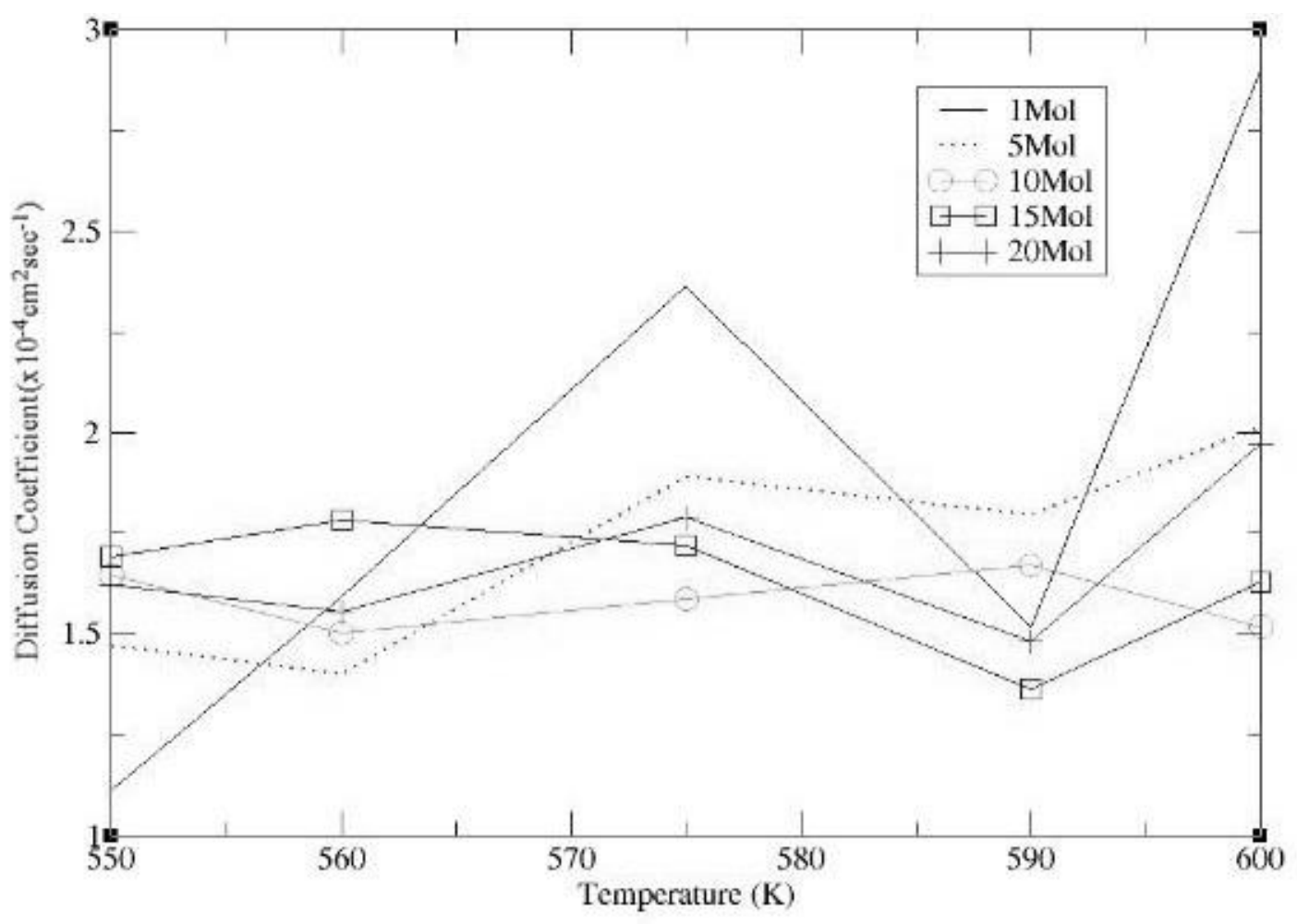

Figure 5. Diffusion coefficient of $\mathrm{H}_{2} \mathrm{O}$ in $\mathrm{P} 3 \mathrm{HT}$ vs. temperature.

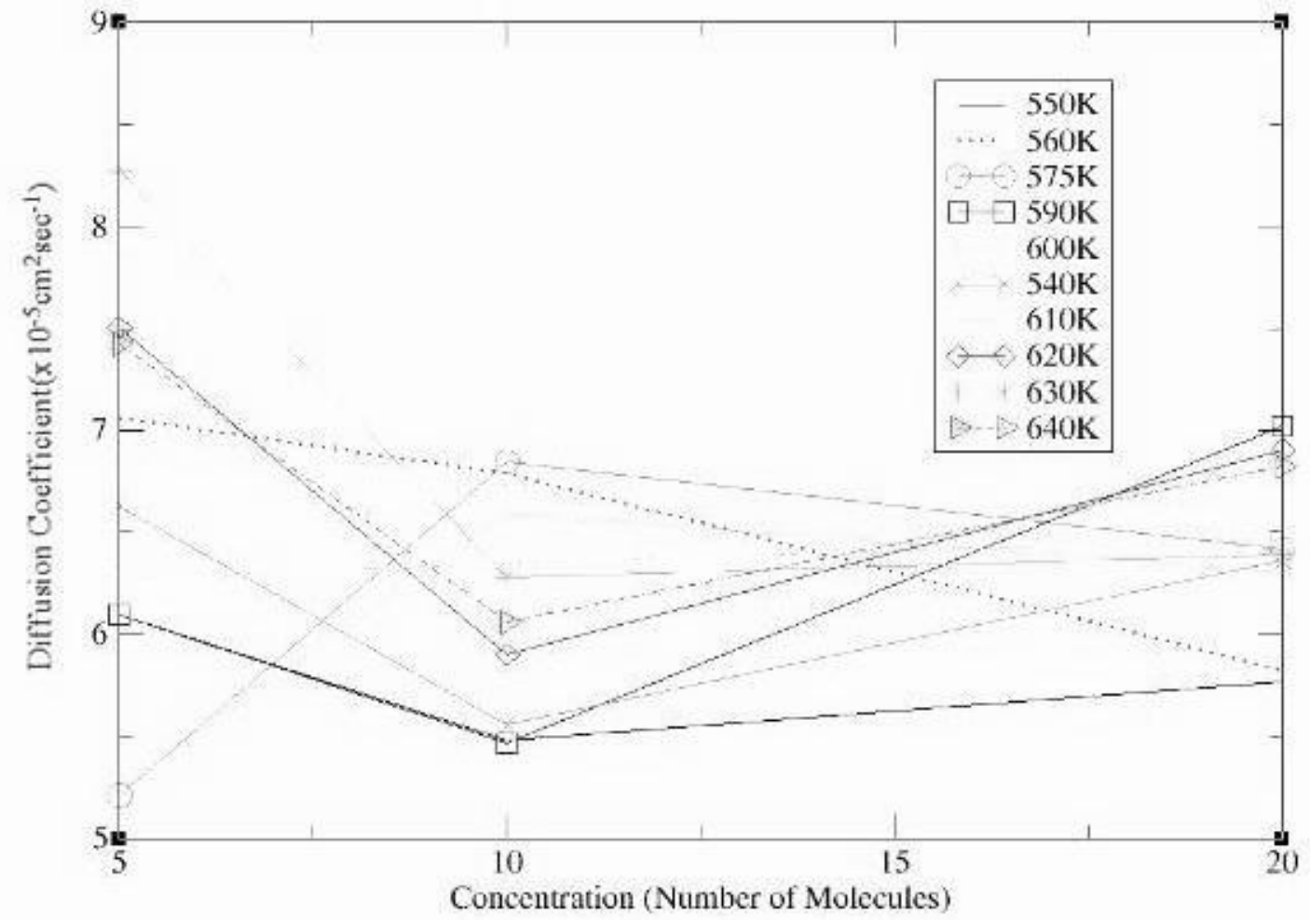

Figure 6. Diffusion coefficient of $\mathrm{O}_{2}$ vs. concentration at 5, 10, and 20 molecules in P3HT. 


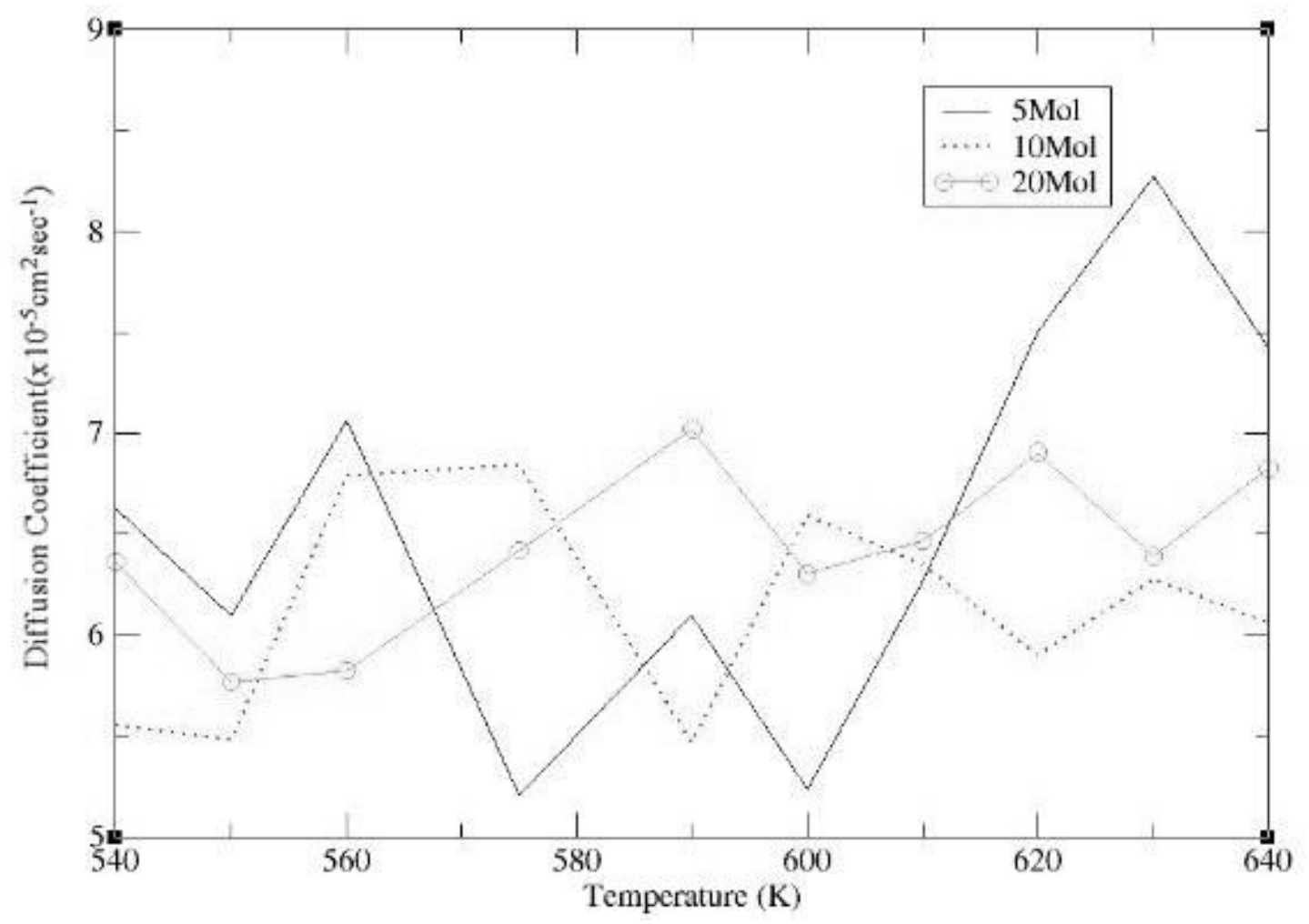

Figure 7. Diffusion coefficient of $\mathrm{O}_{2}$ in $\mathrm{P} 3 \mathrm{HT}$ vs. temperature.

Diffusion coefficient was then analyzed as a function of temperature, shown in figure 7 . Figure 7 shows that the diffusion coefficient does not increase with temperature, which is not what was expected, but matched what was seen with the diffusion of water. Again, the P3HT dymanics possibly could not be changing much in this temperature window so dependence on temperature would not be found.

\section{c. $\mathrm{H}_{2} \mathrm{O}$ with $\mathrm{O}_{2}$ diffusion in $\mathrm{P} 3 \mathrm{HT}$}

As mentioned earlier, one experimental study found that in the presence of water, the diffusion of oxygen would increase in crystalline $\mathrm{P} 3 \mathrm{HT}$. This provided motivation to look at a system of both water and oxygen and see the impacts on the diffusion coefficients for both. 20 molecules of both $\mathrm{H}_{2} \mathrm{O}$ and $\mathrm{O}_{2}$ were placed in the P3HT melt to determine if the molecules would impact each others diffusion. Tables 1 and 2 show the change in diffusion coefficients for both.
The diffusion coefficient of oxygen decreased slightly in the company of water. Water's diffusion coefficient decreased by a factor between four and five with the introduction of oxygen. These results differ from the results obtained in the experimental study that observed humidity increased diffusion of oxygen in crystalline $\mathrm{P} 3 \mathrm{HT}^{2}$. An explanation for the difference has not been reached yet, but the topic is still open for investigation.

It appeared that the oxygen diffused slightly slower, but the water diffused much slower to the speed of the oxygen. The decrease in diffusion coefficient was expected because when more molecules are introduced to a system, they will not be able to move as freely. However, the magnitude of decrease in diffusion coefficient of water was not expected. The change suggested clustering transpired between the water and oxygen molecules. Visual simulations were done to determine if any clustering could be seen. No clustering occurred so that could not be an explanation for the dramatic shift in the diffusion 


\begin{tabular}{|c|c|c|}
\hline Temperature (K) & Without $\mathrm{O}_{2}\left(\times 10^{-4} \mathrm{~cm}^{2} \mathrm{sec}^{-1}\right)$ & With $\mathrm{O}_{2}\left(\times 10^{-4} \mathrm{~cm}^{2} \sec ^{-1}\right)$ \\
\hline 550 & 16.2278 & 4.02382 \\
\hline 575 & 17.8832 & 4.90925 \\
\hline 600 & 19.7319 & 3.98832 \\
\hline
\end{tabular}

Table 1. Diffusion coefficient values for 20 molecules of $\mathrm{H}_{2} \mathrm{O}$ with and without the presence of $\mathrm{O}_{2}$.

\begin{tabular}{|l|l|l|}
\hline Temperature $(\mathrm{K})$ & Without $\mathbf{H}_{2} \mathbf{O}\left(\mathbf{x 1 0}^{-5} \mathbf{c m}^{2} \mathbf{s e c}^{-1}\right)$ & With $\mathbf{H}_{2} \mathbf{O}\left(\mathbf{x 1 0} \mathbf{c m}^{\mathbf{- 5}} \mathbf{s e c}^{-1}\right)$ \\
\hline $\mathbf{5 5 0}$ & 5.76674 & 5.02624 \\
\hline $\mathbf{5 7 5}$ & 6.41666 & 4.75737 \\
\hline $\mathbf{6 0 0}$ & 6.30402 & 4.64397 \\
\hline
\end{tabular}

Table 2. Diffusion coefficient values for 20 molecules of $\mathrm{O}_{2}$ with and without the presence of $\mathrm{H}_{2} \mathrm{O}$.

coefficient of water. The $\mathrm{O}_{2}$ placed in the water, P3HT system had no partial charges which means hydrogen bonding is of no concern. The phenomonon will continue to be researched.

\section{CONCLUSIONS}

Simulations of water and oxygen into a P3HT melt were analyzed to find diffusion coefficient values. The diffusion of both water and oxygen in a P3HT melt are independent of both concentration and temperature in the ranges specified. Diffusion is dependent on the position of the molecule in the $\mathrm{P} 3 \mathrm{HT}$ melt, but with averaging, the diffusion coefficients will converge to a constant value. No trends could be detected with temperature, even when the range was widened.

The diffusion behavior of water and oxygen in the $\mathrm{P} 3 \mathrm{HT}$ melt changes when in the presence of one another. Water drastically slows its diffusion to comparable values for those of oxygen with no observable clustering of the small molecules.

\section{AKNOWLEDGEMENTS}

We thank National Science Foundation, Goodyear, and Lubrizol for financial support.

\section{REFERENCES}

1. Richard Smalley, Energy and Nanotechnology Conference, Rice University, Houston, TX. May 3, 2003.

2. Holger Hintz, H.-J. Egelhaaf, Larry Ler, Jens Hauch, Heiko Peisert, Thomas Chass, Photodegradation of P3HT-A Systematic Study of Environmental Factors. Chemistry of Materials 201123 (2), 145-154.

3. Huang, D. M.; Faller, R.; Do, K.; Moul'o, Characterization of polymer-fullerene mixtures for organic photovoltaics by systematically coarse-grained molecular simulations. A. J. J. Chem. Theory Comput. 2010, 6, 526.

4. H. J. C. Berendsen, ${ }^{*}$ J. R. Grigera,t and T. P. Straatsma, The Missing Term in Effective Pair Potentials. J. Phys. Chem. 1987, 91, 6269-6271.

5. Gaurav Arora and, Stanley I. Sandler, Mass Transport of $\mathrm{O} 2$ and $\mathrm{N} 2$ in Nanoporous Carbon (C168 Schwarzite) Using a Quantum Mechanical Force Field and Molecular Dynamics Simulations. Langmuir 200622 (10), 4620-4628.

6. S. Plimpton, Fast Parallel Algorithms for Short-Range Molecular Dynamics, J Comp Phys, 117, 1-19 (1995). http://lammps.sandia.gov

7. William Humphrey, Andrew Dalkeand Klaus Schulten. Journal of Molecular Graphics, 14:33-38, 1996. 